\title{
The force applied to the knee extensor mechanism differs between flat-footed and normal subjects during walking
}

\author{
Mohsen Razeghi ${ }^{1,2}$, Samaneh Ebrahimi ${ }^{1,2}$, Farzaneh Yazdani ${ }^{1,2,3}$, Behdad Tahayori $^{4}$ \\ 1 Department of Physical Therapy, School of Rehabilitation Sciences, Shiraz University of Medical Sciences, Shiraz, \\ Iran; ${ }^{2}$ Rehabilitation Sciences Research Center, Shiraz University of Medical Sciences, Shiraz, Iran; ${ }^{3}$ Student Research \\ Committee, Shiraz University of Medical Sciences, Shiraz, Iran; 4 Department of Biomedical Engineering, Florida \\ International University, Miami, USA
}

\section{Summary}

Study aim: There is a lack of evidence to show the presence or absence of a relationship between foot morphology and changes of the force applied to the knee extensor mechanism. The purpose of this study was to examine whether the type of foot is a determining factor in the force applied to the extensor mechanism during walking.

Materials and methods: Twenty female subjects (18-30 years), 10 with neutrally aligned feet and 10 with functional flat foot, participated in this study. Data were collected by employing a three dimensional motion capture system and a force platform, while the subjects were walking at their preferred speed. Knee extensor mechanism force was measured at sub-phases of gait (heel strike and toe-off).

Results: A significant interaction was found between groups and sub-phases of gait for all the variables tested. The subjects with flat foot exhibited a significantly higher extensor mechanism force at toe-off compared to the control group ( $\mathrm{p}<0.05$ ).

Conclusion: It can be concluded that subtalar hyper-pronation would increase the force applied to the knee extensor mechanism at toe-off, through increasing the knee sagittal angle, net external flexion moment and extensor mechanism moment arm. Therefore it may increase the possibility of musculoskeletal injuries.

Key words: Flat foot - Motion analysis - Knee extensor mechanism

\section{Introduction}

The unique and specific structure of the foot creates a dynamic link between the ground and the human body, rendering it perfect for bipedal locomotion [24] and ideal for adjusting to different walking conditions [20]. Different mechanisms have been described for such a dynamic body-environment interaction.

The subtalar joint (STJ) complex permits the foot to act as a flexible structure (by pronation) to adapt to uneven surfaces and as a rigid structure (by supination) to facilitate the transmission of forces during gait $[17,19]$. Evidence indicates that during a closed kinematic chain movement (such as the stance phase of walking), the frontal plane motion of the hind limb (supination/pronation) is transmitted to the tibial bone, producing a transverse (external/internal rotation) movement in the shank [22]. Due to its oblique axis, foot pronation would be induced by
STJ directly after initial contact with the ground during normal walking $[11,18,19]$. This maneuver is suggested to be an effective mechanism for shock absorption and foot accommodation in the loading response phase of gait to different terrains [7].

Additionally, the induced internal rotation of the tibia, during STJ pronation, is shown to be coupled with the knee flexion [23]. It has been stated that any factor affecting the foot's normal function may disturb this coupling mechanism [5]. In the flat foot condition, changes in the ankle joint kinetics and kinematics as well as the electromyographic activity of ankle joint muscles have been reported [10]. However, less is known about the consequences of these alterations for the knee joint kinetics. From a clinical perspective, it seems that the alteration in the knee extensor mechanism could increase the risk of soft tissue injury. Providing such a linkage could be clinically relevant during gait analysis procedures and might be used as a biomarker for assessment/prediction of knee joint impairment. 
Therefore, the aim of this study was to examine whether the type of foot is a determining factor in the force applied to the extensor mechanism during walking.

Here, we used the knee extensor mechanism as a measure of force distribution around the knee joint. This simple method utilizes kinematic-kinetic data to measure the extensor mechanism force. We hypothesized that the subjects with subtalar hyper-pronation would demonstrate a greater extensor mechanism force during the stance phase of gait in comparison to subjects with neutrally aligned feet.

\section{Material and methods}

\section{Participants}

Twenty asymptomatic female subjects (aged 18-30 years), 10 with neutrally aligned and 10 with functional flat foot type, were selected after a complete lower extremity clinical examination. Prior to participation, all the subjects were informed about the nature of the study and signed the informed consent form, approved by the Human Ethics Committee of Shiraz University of Medical Sciences.

Flat foot was determined by measuring the resting calcaneal stance position (RCSP) in the frontal plane during weight bearing, using the protocol described by Kim et al. [14]. This measurement has been shown to have high intra- and inter-rater reliability [21]. Having an RCSP between $2^{\circ}$ of inversion and $2^{\circ}$ of eversion was representative of a neutrally aligned foot, while a flat foot presented with an RCSP of more than $4^{\circ}$ of eversion [1].

Also, the Feiss line test was used to define subjects with flexible flat foot. The Feiss line is defined as a straight line from the medial malleolus through the navicular bone to the center of the first metatarsal head, the test being performed during rest and in weight-bearing conditions [8]. Flat feet were labeled as flexible if the navicular bone was positioned under the line only in weight-bearing condition [9].

The inclusion criteria for participants included having a normal range of motion of hip, knee, ankle and metatarsophalangeal joints (based on goniometric assessments), normal (grade five) strength in major lower extremity muscles (as manifested by manual muscle testing performed by the same examiner), having bilateral flat foot (for the patient group, $\mathrm{n}=10$ ) or natural alignment (for the control group, $\mathrm{n}=10$ ). The exclusion criteria were functional or structural orthopedic disorders that would prevent the normal stance phase of walking, such as a limb length discrepancy greater than $1 \mathrm{~cm}$, excessive knee hyper-extension, abnormal knee varus or valgus, chronic pain due to structural or functional problems in the lower extremity bones, ligaments or menisci, neurological ailments affecting the gait such as neuropathy or other sensory disturbance, cerebral palsy, stroke, neurodegenerative disease or any past history of injuries or orthopedic surgery of the lower extremities. All the objective measurements of the study were performed by the same experimenter.

\section{Measure and procedures}

Ground reaction force (GRF) data were collected using a single force plate (Kistler Instrument, Winterthur, Switzerland) with sampling at $240 \mathrm{~Hz}$. Kinematic data were collected using an eight-camera motion analysis system (Proreflex, Qualisys Track Manager Ltd., Gothenburg, Sweden) with sampling at $120 \mathrm{~Hz}$.

To measure the anthropometric data and subsequently build a 6-degree of freedom model, retro-reflective calibration markers of $19 \mathrm{~mm}$ diameter were placed on the following anatomical points: the highest point of the iliac crests, anterior and posterior iliac spines, the center of greater trochanters, dominant lower extremity's medial and lateral femoral condyles, medial and lateral malleoli, first and fifth metatarsal heads, fifth metatarsal base and the center of the calcaneus. Two sets of cluster markers, containing 4 tracking markers secured on a polyform material, were placed on the lateral distal one third of the shank and on the lateral one half of the thigh to track the movement of desired segments [3, 25]. Subjects stood on the force plate and assumed a normal posture for a few seconds to capture a static trial for the sake of model building.

Following multiple practice trials, three barefoot walking trials at a self-selected speed were recorded. To promote a natural gait pattern, subjects were instructed to maintain visual contact with a fixed point located at the end of the walkway at their eye level. Only the trials in which the subject's dominant foot landed on the force plate were considered for further analysis. The dominant leg was determined based on the research done by De Ruiter et al. [6].

Data were synchronously recorded with QTM software (Qualisys Track Manager Ltd., Gothenburg, Sweden). All subsequent analyses were performed in Visual 3D software (C-Motion, Inc., Rockville, MD, USA).

\section{Data analysis}

Raw data were filtered using a fourth order low-pass Butterworth filter with a cut-off frequency of $6 \mathrm{~Hz}$ for kinematic data and $15 \mathrm{~Hz}$ for kinetic data [13]. The lower extremity was modeled as a rigid, linked-segment system. The standard Newton-Euler method was used to calculate the knee joint angle. Ground reaction force was normalized to subjects' weight $(\mathrm{N} / \mathrm{kg})$.

The extensor mechanism force can be measured from net internal extensor torque $\left(\tau_{e m}\right)$ upon knowing the extensor moment arm [16]:

$$
F_{e m}=\tau_{e m} / d_{e m},
$$


where $F_{e m}$ is the extensor mechanism force and $d_{e m}$ is the extensor mechanism moment arm.

To calculate $d_{e m}$ the following formula was used [27]:

$$
d_{\text {em }}=0.0367 x+3
$$

where $x$ is knee joint angle in degrees. On the other hand

$$
\tau_{\text {knee }}=\mathrm{GRF} * d_{\mathrm{GRF}},
$$

where $\tau_{\text {knee }}$ is knee torque, and $d_{\mathrm{GRF}}$ is the moment arm of GRF. Knee torque was calculated from the model developed in Visual 3D software. Assuming that in a semistatic situation the two torques of the extensor mechanism and knee are equal $\left(\tau_{e m}=\tau_{\text {knee }}\right)[2,13]$, the unknown $\tau_{\text {em }}$ in Eq. (1) can be replaced by knee torque. The extensor mechanism force at heel strike and toe-off was calculated. The average of the knee extensor mechanism force for the whole stance was calculated based on the mean values of knee joint sagittal angle, mean ground reaction force, mean moment arm of GRF and mean moment arm of extensor mechanism.

\section{Statistical analysis}

Prior to data analysis, each of the dependent variables in question was examined for distribution normality with the Kolmogorov-Smirnov test, using the 0.05 level of significance. Each variable demonstrated normality, and as a result, parametric statistics were used.

A $2 \times 2$ (group $\times$ event) split-plot ANOVA model was used to analyze the dependent variables. Subjects were nested within independent groups (control vs. flatfoot) and gait event (heel strike vs. toe-off) was a repeated measures variables. The following dependent variables were analyzed: knee angle, knee moment and force. Given the multicollinearity between the dependent variables, the overall ANOVA was tested at the more conservative 0.01 level. The correlation between resting calcaneal stance position (RCSP) and mean knee extensor mechanism force (average through the stance phase of the gait cycle) was examined using the Pearson correlation.

\section{Results}

Twenty asymptomatic female subjects participated in the study. There were no statistically significant differences in age, height or weight distribution between the groups (Table 1).

For knee angle, a significant interaction was present between groups and test $\left(\mathrm{F}_{1,18}=13.15, \mathrm{p}<0.01\right)$. Followup analysis of this interaction indicated that both the control $\left(t_{9}=29.06, p<0.01\right)$ and flat-foot group $\left(t_{9}=27.60\right.$, $\mathrm{p}<0.01)$ increased the knee angle between heel strike and toe-off, as expected. There was no significant difference between the groups in knee angle at heel strike $\left(\mathrm{t}_{18}=1.46\right.$, $\mathrm{p}=0.160$ ), but there was a significant difference at toe-off between the groups $\left(\mathrm{t}_{18}=5.01, \mathrm{p}<0.01\right)$.

For knee moment, there was also a significant interaction between groups and test $\left(\mathrm{F}_{1,18}=8.81, \mathrm{p}<0.01\right)$. Follow-up analysis of this interaction indicated that both the control $\left(\mathrm{t}_{9}=-4.99, \mathrm{p}<0.01\right)$ and flat-foot group $\left(\mathrm{t}_{9}=-7.76\right.$, $\mathrm{p}<0.01)$ increased the knee moment between heel strike and toe-off, as expected. There was no significant difference between the groups in knee moment at heel strike $\left(\mathrm{t}_{18}=-0.99, \mathrm{p}=0.352\right)$, but there was a significant difference at toe-off between the groups $\left(\mathrm{t}_{18}=-3.76, \mathrm{p}<0.01\right)$.

Finally, for the force applied to the knee extensor mechanism, there was also a significant interaction between groups and test $\left(\mathrm{F}_{1,18}=16.18, \mathrm{p}<0.01\right)$. Followup analysis of this interaction indicated that both the control $\left(\mathrm{t}_{9}=-3.98, \mathrm{p}<0.01\right)$ and flat-foot group $\left(\mathrm{t}_{9}=-7.22\right.$, $\mathrm{p}<0.01)$ increased the force between heel strike and toe-off, as expected. There was no significant difference between the groups in force at heel strike $\left(\mathrm{t}_{18}=-0.96\right.$, $\mathrm{p}=0.350$ ), but there was a significant difference at toe-off between the groups $\left(\mathrm{t}_{18}=-3.69, \mathrm{p}<0.01\right)$.

The Pearson coefficient of determination showed a positive correlation between resting calcaneal stance position (RCSP) and extensor mechanism force $\left(\mathrm{R}^{2}=0.624\right.$, $\mathrm{p}<0.001)$ in the group with flat foot. This determination coefficient was not significant in the normal group

Table 1. The participants' demographic characteristics

\begin{tabular}{lccccc}
\hline & Group & N & Mean \pm SD & Range & p value \\
\hline Age $[$ year] & NL & 10 & $21.76 \pm 3.48$ & $18-30$ & 0.862 \\
& FF & 10 & $21.73 \pm 3.29$ & $18-28$ & \\
Height $[\mathrm{cm}]$ & NL & 10 & $156.36 \pm 4.59$ & $152-168$ & 0.743 \\
Body mass $[\mathrm{kg}]$ & FF & 10 & $156.42 \pm 4.21$ & $153-167$ & \\
& NL & 10 & $58.91 \pm 3.32$ & $55.14-65.01$ & 0.781 \\
\hline
\end{tabular}

$\mathrm{N}$ - number of subjects in each group; SD - standard deviation; NL - normal; FF - flat-footed. 


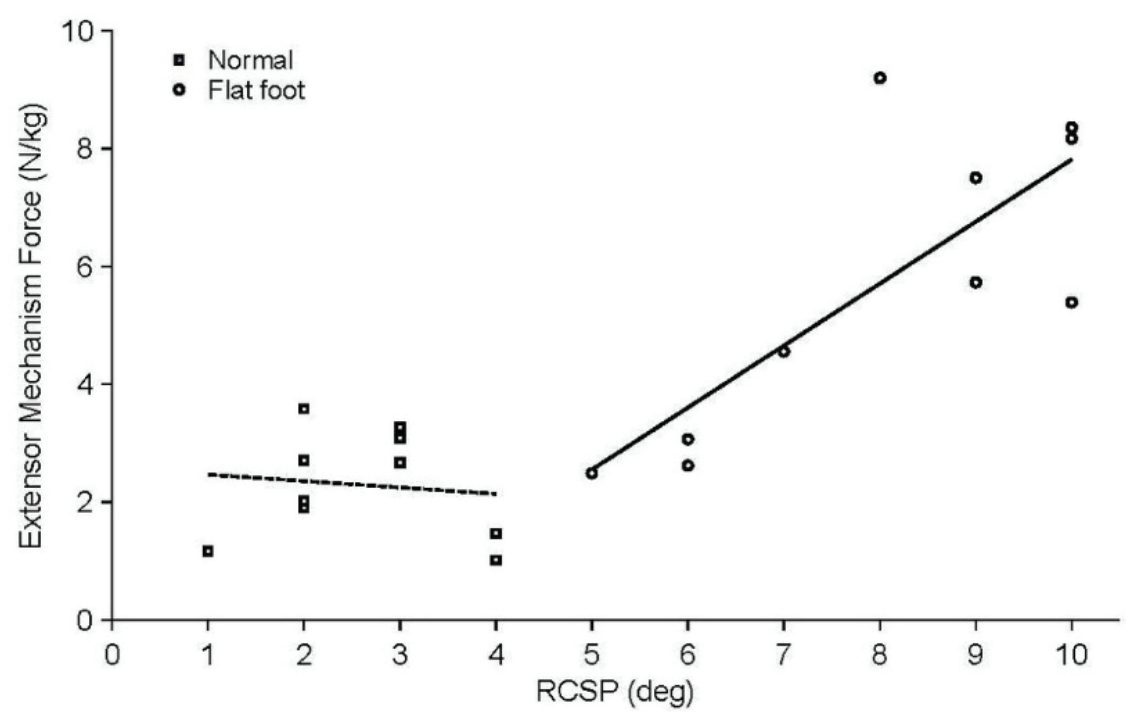

Fig. 1. Relation between ankle valgus angle and mean knee extensor mechanism force

$\left(\mathrm{R}^{2}=0.013, \mathrm{p}=0.846\right)$, suggesting no significant relation between these two parameters in the normal condition (Fig. 1).

Normal subjects had a statistically significantly lower valgus angle $(5.7 \pm 2.5$ vs. $8 \pm 1.9 ; \mathrm{p}<0.001)$. Mean extensor mechanism force was calculated by taking the average of the extensor mechanism force throughout the stance phase. In the healthy group, there was not a significant correlation between RCSP and mean extensor force $\left(\mathrm{R}^{2}=0.013, \mathrm{p}=0.846\right)$. However, in subjects with flat foot, there was a significant positive correlation between the two variables $\left(\mathrm{R}^{2}=0.624, \mathrm{p}<0.001\right)$.

\section{Discussion}

From a biomechanical perspective, the human body has powerful relative synchronous interactions between its segments during walking [12]. Any asynchrony in these motions could potentially result in injury, likely due to an alteration in the knee joint pattern of movement and force distribution. During a normal gait cycle, knee extension must be associated with tibial external rotation about midstance in order to preserve congruency of the joint. In a hyper-pronation situation the femur undergoes excessive internal rotation to maintain relative knee external rotation and compensate for the excessive tibial external rotation [23].

Our data showed a significantly greater knee flexion angle at the end of the stance phase of walking in the group with flat foot. It is suggested that in a closed kinematic chain of lower limb movement, talar adduction induces knee flexion through tibial internal rotation [5]. Our current finding, therefore, is in agreement with this biomechanical chain of effects. Examining the correlation between the degree of valgus and the extensor mechanism force showed that in the pathologic condition an increase in RCSP is linearly related to the extensor mechanism force. Although our study did not pinpoint the cause of alteration in knee joint mechanisms, these current findings strongly suggest that alterations in the knee joint are related to abnormalities of the foot. However, more thorough studies are needed to investigate the nature of the alteration in the extensor mechanism.

The current study analysis showed that at the beginning of the stance phase, both groups demonstrate a similar knee angle and extensor mechanism profile. However, at toe-off, these variables significantly increase in the flatfoot group. These observed deviations could be due to excessive tibial rotation or alteration in forefoot movements. A study by Hunt and Smith (2004) showed that subjects with flat foot have decreased forefoot adduction at terminal stance [10]. However, our study was not designed to investigate the movements of different foot segments and their relation to the knee extensor mechanism.

Insertion of a prosthetic insole, which is believed to compensate for the subtalar hyper-pronation, has been shown to reduce the knee joint flexion angle during walking $[4,15]$. More importantly, it has also been shown that runners with lower arch height exhibit a greater eversion to tibial internal rotation ratio compared to those with high arches [26]. Taken together with our findings, it can be suggested that the observed excessive knee joint angle at toe-off is caused by subtalar hyper-pronation.

Despite its limitations, this study was the first to perform a $3 \mathrm{D}$ analysis of the influence of foot morphology on the extensor mechanism force. A simplified model of the knee joint was used in this study, which ignored the co-contractions from muscles, e.g. hamstring. Therefore the net external flexion moment was not a free muscle moment but, for simplification, was considered to be so in 
this investigation. The determination of anatomical landmarks has always been subject to some errors, and this was a known limitation of our procedure. Moreover, the subjects of this study were all young females who were not representative for flat-footed subjects.

\section{Conclusion}

We found that functional flat foot can alter force distribution as well as joint range of motion of the knee joint during the stance phase of gait. This novel evidence adds to our understanding about the impact of functional flat foot on the knee joint from a biomechanical perspective. From a clinical standpoint, alterations in the moment arm of a muscle could have devastating consequences in the final torque and hence movement generation. An apparently small change in the moment arm (such as $0.18 \mathrm{~cm}$ as observed in this study) can significantly alter the knee joint extensor mechanism. Our study showed that subjects with flat foot demonstrate a significant alteration in the knee extensor mechanism at terminal stance. However, the chain of events which causes such an alteration is yet to be determined. Such a finding could be of important clinical relevance since force redistribution on the knee joint and subsequently abnormal stresses on the soft tissues increase the tendency for musculoskeletal injuries.

\section{Conflict of interest: Authors state no conflict of interest.}

\section{References}

1. Bok S.K., Kim B.O., Lim J.H., Ahn S.Y. (2014) Effects of custom-made rigid foot orthosis on pes planus in children over 6 years old. Ann. Rehabil. Med., 38(3): 369-375.

2. Bressel E. (2001) The influence of ergometer pedaling direction on peak patellofemoral joint forces. Clin. Biomech., 16(5): 431-437.

3. Cappozzo A., Catani F., Della Croce U., Leardini A. (1995) Position and orientation in space of bones during movement: anatomical frame definition and determination. Clin. Biomech., 10(4): 171-178.

4. Chen Y.C., Lou S.Z., Huang C.Y., Su F.C. (2010) Effects of foot orthoses on gait patterns of flat feet patients. Clin. Biomech., 25(3): 265-270.

5. Deleo A., Dierks T., Ferber R. ( 2004) Lower extremity joint coupling during running: a current update. Clin. Biomech., 19(10): 983-991.

6. De Ruiter CJ, De Korte A, Schreven S, De Haan A. (2010) Leg dominancy in relation to fast isometric torque production and squat jump height. Eur. J. Appl. Physiol.,108(2): 247.
7. Duval K., Lam T., Sanderson D. (2010) The mechanical relationship between the rearfoot, pelvis and low-back. Gait Posture, 32(4): 637-640.

8. Feiss HO. (1909) A simple method of estimating the common variations and deformities of the foot. Am. J. Med. Sci., 138(2): 213-230.

9. Hannigan-downs K., Harter R., Smith G. (2000) Radiographic validation and reliability of selected clinical measures of pronation. J. Athlet. Train., 35: 12-30.

10. Hunt A., Smith R. (2004) Mechanics and control of the flat versus normal foot during the stance phase of walking. Clin. Biomech., 19(4): 391-397.

11. Karatsolis K., Nikolopoulos C., Papadopoulos E., Vagenas G., Terzis E., Athanasopoulos S. (2009) Eversion and inversion muscle group peak torque in hyperpronated and normal individuals. The Foot, 19(1): 29-35.

12. Khamis S., Yizhar Z. (2007) Effect of feet hyperpronation on pelvic alignment in a standing position. Gait Posture, 25(1): 127-134.

13. Khan W., Nokes L., Jones R., Johnson D. (2007) The relationship of the angle of immobilisation of the knee to the force applied to the extensor mechanism when partially weight-bearing. Bone Joint J., 89: 911-914.

14. Kim J.A., Lim O.B., Yi C.H. (2015) Difference in static and dynamic stability between flexible flatfeet and neutral feet. Gait Posture, 41(2): 546-550.

15. Leung A., Mak A., Evans J. (1998) Biomechanical gait evaluation of the immediate effect of orthotic treatment for flexible flat foot. Prosthet. Orthot. Int., 22(1): 25-34.

16. Nordin M., Frankel V. (2001) Basic biomechanics of the musculoskeletal system, Philadelphia, Lippincott Williams \& Wilkins.

17. Perry J., Gait analysis: normal and pathological function, SLACK incorporated, 1992.

18. Pohl M., Messenger N., Buckley J. (2007) Forefoot, rearfoot and shank coupling: effect of variations in speed and mode of gait. Gait Posture, 25(2): 295-302.

19. Sammarco G., (1989) Biomechanics of the foot. In: Nordin M., Frankel V., (eds.) Basic biomechanics of the musculoskeletal system: Lea \& Febiger, Philadelphia. p. 163-181.

20. Shariatmadari M., English R., Rothwell G. ( 2010) A review of developments into therapeutic running footwear research. Procedia Eng., 2(2): 2811-2816.

21. Smith-oricchio K., Harris B. (1990) Interrater reliability of subtalar neutral, calcaneal inversion and eversion. $J$. Orthop. Sports Phys. Ther., 12(1): 10-15.

22. Tateuchi H., Wada O., Ichihashi N. (2011) Effects of calcaneal eversion on three-dimensional kinematics of the hip, pelvis and thorax in unilateral weight bearing. Hum. Mov. Sci., 30(3): 566-573.

23. Tiberio D. (1987) The effect of excessive subtalar joint pronation on patellofemoral mechanics: a theoretical model. J. Orthop. Sports Phys. Ther., 9(4): 160-165. 
24. Wang W., Crompton R. (2004) Analysis of the human and ape foot during bipedal standing with implications for the evolution of the foot. J. Biomech., 37(12): 1831-1836.

25. Wilken J.M., Rodriguez K.M., Brawner M., Darter B.J. (2012) Reliability and minimal detectible change values for gait kinematics and kinetics in healthy adults. Gait Posture, 35(2): 301-307.

26. Williams D.S., Mcclay I.S., Hamill J., Buchanan T.S. (2001) Lower extremity kinematic and kinetic differences in runners with high and low arches. J. Appl. Biomech., 17(2): 153-163.

27. Yamaguchi G., Zajac F. (1989) A planar model of the knee joint to characterize the knee extensor mechanism. J. Biomech., 22(1): 1-10.

\section{Received 30.01.2018 \\ Accepted 02.07.2018}

(C) University of Physical Education, Warsaw, Poland

\section{Acknowledgments}

The present article was financially supported by Shiraz University of Medical Sciences grant No. 87-4219. We thank Dr. Nasrin Shokrpour at Research Consultation Center of Shiraz University of Medical Sciences for editorial assistance. 